\title{
Forced dewetting on porous media
}

\section{By OLIVIER DEVAUCHELLE, CHRISTOPHE JOSSERAND and STEPHANE ZALESKI}

Laboratoire de Modélisation en Mécanique, CNRS-UMR 7606, Case 162, 4 place Jussieu, 75252 Paris Cédex 05-France

(Received 13 November 2018)

We study the dewetting of a porous plate withdrawn from a bath of fluid. The microscopic contact angle is fixed to zero and the flow is assumed to be parallel to the plate (lubrication approximation). The ordinary differential equation involving the position of the water surface is analysed in phase space by means of numerical integration. We show the existence of a critical value of the capillary number $\eta U / \gamma$, above which no stationary contact line can exist. An analytical model, based on asymptotic matching is developed, that reproduces the dependence of the critical capillary number on the angle of the plate with respect to the horizontal for large control parameters (3/2 power law).

\section{Introduction}

When sea retreats from the shore, sand structures appear as solid granular particles are transported via the liquid. Liquid motion and particularly film retraction on an erodible medium are known to create impressive erosion patterns, such as sand ripples for oscillatory waves (Stegner \& Wesfreid 1999; Scherer et al. 1999) or sand furrows (Daerr et al. 2003; Schorghofer et al. 2004). The case of liquid retraction from a granular bed can be understood as a dewetting dynamics on a porous erodible bed. Such physical phenomena have been reproduced in the laboratory by pulling a plate covered with a bed of grains out of a liquid tank (Daerr et al. 2003). This situation is similar to the well-known experiment investigating a moving contact line on a non-porous plate (Blake \& Ruschak 1979). In this latter case, a contact line exists for small removal speed $U$, whereas for higher speed (above a well-defined critical value $U_{c r}$ ) a macroscopic water film (the so-called LandauLevich-Derjaguin film (denoted LLD later on), see Landau \& Levich (1942); Deriagin (1943)) covers the whole plane (Eggers 2004a). We propose here to investigate this transition for a saturated porous medium, in connection with recent experiments involving granular materials by Daerr et al. (2003). There, a motor-driven plane, covered with a granular layer, is withdrawn from a water tank at constant speed $U$. The solid plane is tilted to an angle $\theta$. At high enough velocity, erosion river networks and mudflows are observed, whereas only light patterns appear at smaller speed. We investigate the loss of a static contact line and seek to relate it to the transition between various erosion regimes. We therefore seek the critical velocity above which no static contact line can exist on a granular bed. Below this critical velocity, almost no grain motion is observed so that we identify the granular bed with a rigid porous medium. Dewetting on a porous medium has already been studied in different configurations (see Raphaël \& de Gennes (1999); Aradian et al. (2000); Bacri \& Brochard-Wvart (2001) and references herein). The case of a porous plate removed from a liquid was studied by Raphaël \& de Gennes (1999), but the focus was on the spatial evolution of the LLD film, the existence of which 


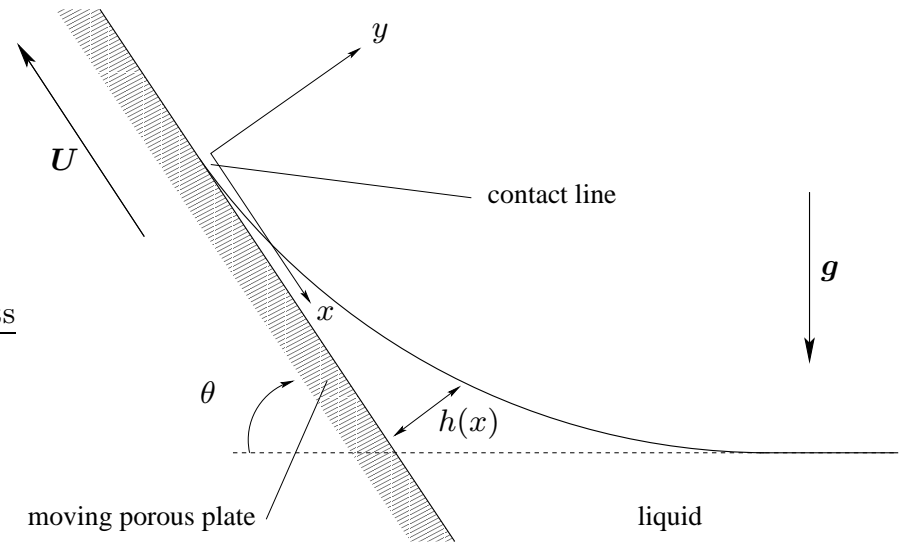

Figure 1. A porous plate of conductivity $k$ is being withdrawn from a liquid bath with speed $\mathbf{U}$ at angle $\theta$.

was assumed. The contact line dynamics was also studied (Aradian et al. 2000) for a wet horizontal support in which the liquid is sucked in the dry porous medium.

From a more theoretical point of view, the problem of a moving contact line on a porous solid is pointed out by de Gennes (1985) as a natural regularization for the contact line dynamics equations. The bulk liquid flow through the porous solid indeed removes the usual stress singularity that one would encounter at a contact line with a no-slip condition (Dussan \& Davis 1974). No additional assumption, such as the introduction of a Navier slip at microscopic scale, is then required. However, another question arises when considering a porous medium: what is the relevant condition for the contact angle at the contact line? As discussed below, we propose here for a saturated system to take a zero contact angle.

The paper is organized as follows: in the next Section, we use the lubrication approximation to deduce the equation for the static interface shape, both for the contact line and a zero-flux LLD film. In Section 3 we exhibit the transition between these two configurations as the pulling velocity increases by means of a shooting method. Then, we propose to interpret the solutions in the framework of dynamical systems (Section 44).

\section{Principles}

\subsection{Lubrication approximation}

Our approach seeks to determine the velocity (if any) above which the static contact line can no longer exist in a granular bed withdrawal experiment. Below this velocity, we can consider that the grains almost do not move relatively to the withdrawn plate. Thus the granular material is represented by a non-erodible porous medium (see Figure 11) of permeability $k$, and we only have to investigate the stationary problem. The fluid is characterized by its density $\rho$, dynamical viscosity $\eta$ and surface tension $\gamma$. Assuming invariance in the $z$-direction, we consider the two-dimensional problem where the water surface is described by the function $h(x)$. For the plate velocities pertinent to the problem (typically $\left.0.5 \mathrm{~cm} \cdot \mathrm{s}^{-1}\right)$ and the estimated porosity of the granular bed $\left(k \approx 10^{-12} \mathrm{~m}^{2}\right)$ we can consider that the porous medium remains fully saturated with water at any distance from the free water level.

We will restrict our analysis to small angles $\theta$ so that the lubrication approximation can be employed (i.e. $\theta,\left\|h^{\prime}\right\| \ll 1$ where the ' stands for the $x$-derivative). Only the 
$x$-component $u$ of the velocity has to be taken into account, for which Poiseuille profile is assumed, with a vanishing tangential stress on the gas side:

$$
\left.\frac{\partial u}{\partial y}\right|_{y=h(x)}=0 .
$$

Another boundary condition has to be written at the porous surface. The classical noslip condition, as required at the solid-fluid interface on an impermeable plate, leads to the following equation for $h(x)$ (its derivation is similar to the one presented in Appendix B):

$$
h^{\prime \prime \prime}-h^{\prime}+\theta=\frac{3 \mathrm{Ca}}{h^{2}},
$$

where lengths $h$ and $x$ have been made dimensionless by the capillary length $l_{c}=$ $\sqrt{\gamma / \rho g}\left(l_{c} \approx 2.8 \mathrm{~mm}\right.$ for water) and Ca is the capillary number, defined by

$$
\mathrm{Ca}=\frac{\eta U}{\gamma}
$$

Equation (2.2) and any derivative of its solutions are singular at the contact line, where $h=0$ (Duffy \& Wilson 1997). For a non-porous surface, a short-length regularization is invoked coming either from effective slip near the contact line (Huh \& Scriven 1971), the existence of a pre-wetting liquid film and Van der Waals forces (de Gennes 1984; Hervet \& de Gennes 1984) or a "diffuse interface model" (Seppecher 1996). Such a regularization always involves a microscopic cut-off length (on the order of $1 \mathrm{~nm}$ ) below which it is claimed that hydrodynamics fails. The Navier slip condition is then mostly used in numerical simulations investigating moving contact line problems (Renardv et al. 2001). This condition reads at the solid-fluid interface: $u-U=\Lambda_{N} \partial u / \partial y$ at $y=0$ where $\Lambda_{N}$ is the cut-off length. If $\lambda_{N}$ is the rescaled cut-off length (that is $\lambda_{N}=\Lambda_{N} / l_{c}$ ), Equation (2.2) becomes

$$
h^{\prime \prime \prime}-h^{\prime}+\theta=\frac{\mathrm{Ca}}{h^{2} / 3+\lambda_{N} h} .
$$

Equation (2.3) can be numerically solved and analytically approached. A contact line is then found to exist as long as the capillary number is smaller than a critical value, above which a macroscopic LLD film is deposited on the solid (Eggers 2004a). Notice however that not all the singularities discussed above are suppressed by the Navier slip condition since the capillary pressure still diverges at the contact line (see Appendix (A).

\subsection{The case of porous solid}

A porous solid allows for both interfacial slip (first proposed by Beavers \& Joseph (1967)) and bulk flow. Using the Brinkman equation to describe the flow inside the porous medium, Neale \& Nader (1974) showed that, for a homogeneous porous media, the magnitude of the slip is proportional to the prevailing shear stress:

$$
\left.u\right|_{y=0}-\left.u_{p}\right|_{y=0}=\left.\frac{\sqrt{k}}{\alpha} \frac{\partial u}{\partial y}\right|_{y=0}
$$


where $k$ is the permeability of the solid, $\alpha$ a coefficient of order one, and $u_{p}$ the velocity of the fluid in the porous medium. Darcy's law holds in the solid so that

$$
u_{p}+U=-\frac{k}{\eta}\left(\frac{\partial p}{\partial x}-\rho g \theta\right)
$$

and finally the Equation hereafter describes the shape of the steady fluid film under withdrawal (the detailed derivation is presented in Appendix $\mathbb{B}$ ):

$$
h^{\prime \prime \prime}-h^{\prime}+\theta=\frac{\mathrm{Ca}}{h^{2} / 3+\lambda h / \alpha+\lambda^{2}},
$$

where $\lambda=\sqrt{k} / l_{c}$. Such an equation is similar to those studied for the contact line on a solid surface, using specific boundary conditions at the solid surface (Eggers 2004a; Hocking 2001). It has been shown (in the case of a plate pushed into water) that the details of the regularization do not influence the far-field fluid flow as long as the cut-off length is small enough (Eggers 2004b). However, an important difference in our case lies in the typical values of $\lambda$ involved in porous media $\left(\approx 10^{-2}\right.$ in Daerr et al. $\left.(2003)\right)$ to be compared with $10^{-6}$ for regular solids.

Here we would like to point out that in recent papers (Maurer et al. 2003; Hadiconstantinou 2003 ) a second order-slip law is used to model the flow of gases at large Knudsen numbers. This boundary condition (adapted to the present notations) reads

$$
\left.u\right|_{y=0}+U=\left.\Lambda_{C} \frac{\partial u}{\partial y}\right|_{y=0}-\left.\alpha_{C} \Lambda_{C} \frac{\partial^{2} u}{\partial y^{2}}\right|_{y=0},
$$

where $\alpha_{C}$ is a positive coefficient of order one, and $\Lambda_{C}$ is a slip length of the same order than the mean free path of the gas. If such a boundary condition were used in the case of a contact line, again Equation (2.6) would be obtained.

\subsection{Boundary conditions}

The limit for large positive values of $x$ is well-defined: the water surface is horizontal far from the plane, that is

$$
h(x) \underset{x \rightarrow \infty}{\sim} \theta x .
$$

For the two remaining boundary conditions, two different cases will be studied, depending on whether a contact line is formed between the water surface and the solid plate, or if a film of water remains on the solid surface. In the first case, the water level vanishes at the origin and a contact angle $\theta_{0}$ is usually imposed; the contact line set of boundary conditions is

$$
\left\{\begin{aligned}
h(0) & =0 \\
h^{\prime}(0) & =\theta_{0} .
\end{aligned}\right.
$$

Following Raphaël \& de Gennes (1999), we will hereafter consider that the contact angle $\theta_{0}$ is zero for dewetting on a porous media. We argue indeed that for saturated porous media, the liquid film wets completely the surface, leading to an effective zero contact angle.

When LLD film starts at the meniscus, the only boundary condition known a priori is

$$
\lim _{x \rightarrow-\infty} h(x)=h_{f}
$$


where $h_{f}$ is a constant solution of Equation (2.6). Notice that Equation (2.6) stands only for zero-flux films, that neither add nor withdraw water from the tank. We omit the important case where a LLD film is continuously growing with time, a case that was investigated by Hocking (2001). Our study is therefore relevant to determine the loss of a static contact line solution, without any information about the dynamics. The stability of the solution as well as the time-dependent dynamics of a moving meniscus cannot be studied at this stage and will be the purpose of further work. However, we will see in Section 4 that the film solutions of (2.6) satisfying (2.9) play an important role in the dynamical system describing our solutions. Finally, for a solution to be acceptable, the water level must always lay above the porous medium: $\forall x, h(x)>0$.

\subsection{Parameters}

The parameter $\alpha$ comes from the detailed modelisation of the interface slip flow (Beavers \& Joseph 1967; Goveau et al. 2003), and varies generally between 0.1 and 4 (Neale \& Nader 1974). Notice for instance that if $\alpha$ is smaller than $\sqrt{3} / 2$, Equation (2.6) becomes singular for some negative values of $h$. However, for the sake of simplicity the coefficient $\alpha$ is fixed to one in the present study. Now, for $\alpha=1$, Equation (2.6) is an ordinary differential equation (ODE) with three parameters: $\theta, \mathrm{Ca}$ and $\lambda$. In fact, one should notice that this equation is only a two-parameter ODE: defining $h_{*}=h / \lambda$, Equation (2.6) becomes

$$
h_{*}^{\prime \prime \prime}-h_{*}^{\prime}+\theta^{*}=\frac{\mathrm{Ca}^{*}}{h_{*}^{2} / 3+h_{*}+1},
$$

where $\theta^{*}$ and $\mathrm{Ca}^{*}$ are defined as follow:

$$
\begin{gathered}
\theta^{*}=\theta / \lambda=\theta \sqrt{\frac{\gamma}{\rho g k}}, \\
\mathrm{Ca}^{*}=\mathrm{Ca} / \lambda^{3}=\frac{U \eta \sqrt{\gamma}}{(\rho g k)^{3 / 2}} .
\end{gathered}
$$

Equation (2.10) is the one we will study later on, but we will omit the ${ }_{*}$ on $h_{*}$ for the sake of readability. These nondimensional parameters were choosen because they are proportional to the two experimental parameters that may be easily and continuously tuned, namely $U$ and $\theta$. If the permeability of the porous solid is extremely low, both $\theta^{*}$ and $\mathrm{Ca}^{*}$ tend to infinity, as well as the ratio $\mathrm{Ca}^{*} / \theta^{*}=U \eta /(\theta \rho g k)$. In this case, the velocity inside the porous medium is extremely slow compared to $U$. For the experimental study of Daerr et al. (2003), the rescaled capillary number $\mathrm{Ca}^{*}$ is of the order of $10^{5}$.

\subsection{Hydrostatic solutions}

Any solution which respects the boundary condition (2.7) for large $x$ verifies

$$
\lim _{x \rightarrow+\infty} h(x)=+\infty,
$$

thus for large $x$, Equation (2.10) becomes

$$
h^{\prime \prime \prime}-h^{\prime}+\theta^{*}=0 .
$$

The behaviour of the water surface at large $x$, hereafter denoted by $h_{\infty}$, is directly obtained from Equation (2.11):

$$
h_{\infty}(x)=A_{\infty}+\theta^{*} x+\left(\theta^{*}-\theta_{\mathrm{ap}}^{*}\right) \exp (-x),
$$




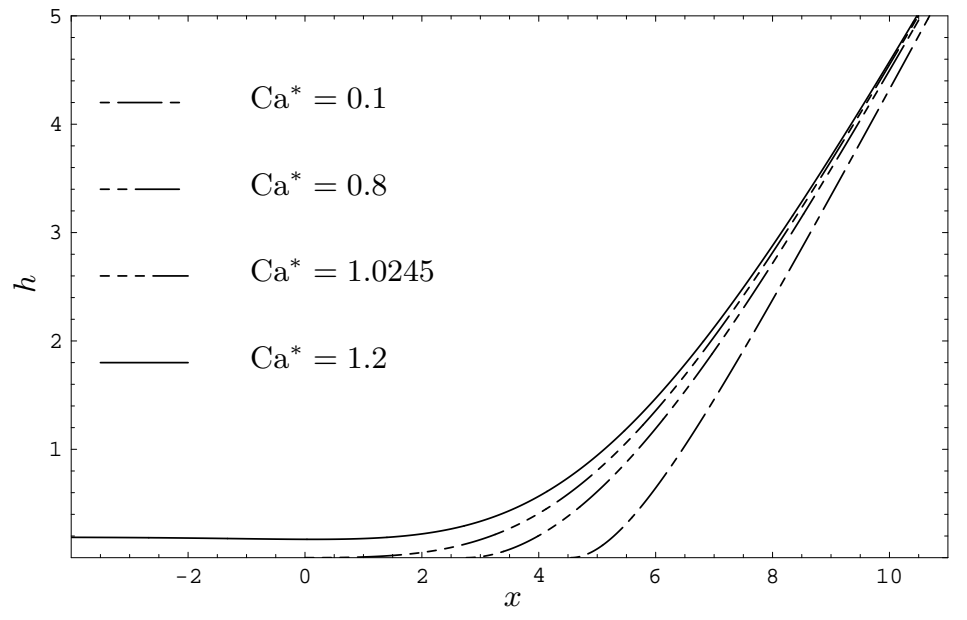

Figure 2. Numerical solutions of Equation (2.10) for different capillary numbers. The rescaled tilt angle $\theta^{*}$ is fixed to 1

where $A_{\infty}$ and $\theta_{\text {ap }}^{*}$ are two constants, corresponding respectively to the length of the dynamical meniscus and to the so-called apparent contact angle (note that the dimensional apparent contact angle is actually $\theta_{\mathrm{ap}}^{*} \lambda$ ). Even though the lubrication approximation is not expected to hold for large $x$ since the water level is not small anymore, Equation (2.11) leads to the classical static meniscus solution (remember that $x$ has been scaled by the capillary length $l_{c}$ ). Consequently, we may consider that Equation (2.10) holds at any position on the $x$-axis.

\section{Numerical results}

\subsection{Contact line solutions}

To seek steady contact line solutions, Equation (2.10) may be solved numerically using a finite-difference algorithm. In the case of a contact line, two boundary conditions may be fixed at $x=0$ by the contact line conditions (2.8). The third condition comes from the flat water level at infinity (2.7). With the new notations, we end up with the following system:

$$
\begin{gathered}
h^{\prime \prime \prime}-h^{\prime}=f(h), \\
h(0)=h^{\prime}(0)=0, \\
h(x) \underset{x \rightarrow \infty}{\sim} \theta^{*} x,
\end{gathered}
$$

where

$$
f(h)=\frac{\mathrm{Ca}^{*}}{h^{2} / 3+h+1}-\theta^{*} .
$$

We use a shooting method (see Manneville (1990)), varying the initial curvature $h^{\prime \prime}(0)$ in order to find the numerical solution which corresponds to the hydrostatic condition at large $x$. Some numerical contact line solutions to Equation (3.1) are presented on Figure 2 for different $\mathrm{Ca}^{*}$ at fixed $\theta^{*}$. 


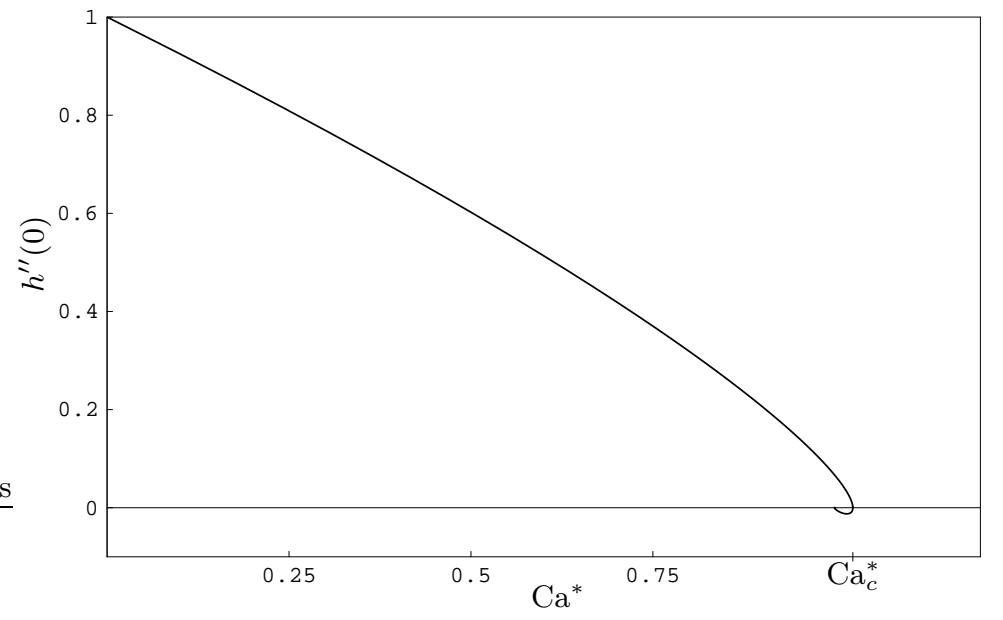

Figure 3. Curvature at the origin versus rescaled capillary number, for a contact line solution of Equation 2.10] The rescaled tilt angle $\theta^{*}$ is fixed to 1 . No solution is found for capillary numbers higher than $\mathrm{Ca}_{c}^{*} \approx 1.0247$.

As shown on Figure 2 the contact line zone is somehow streched as the capillary number is increased. In other words, the curvature $h^{\prime \prime}(0)$ at the origin tends to zero as $\mathrm{Ca}^{*}$ tends to a critical value $\mathrm{Ca}_{c}^{*}$. Above this critical value, no contact line solution can be found by the shooting mehod. This transition will be clarified below using the dynamical system associated to (3.1). The disappearance of the contact line solution may be represented in a kind of bifurcation diagram, plotting the curvature at the origin against the capillary number, as shown in Figure 3 Notice that, even though the curvature at the origin tends to zero as $\mathrm{Ca}_{c}^{*}$ is approached, the contact line solution does not become unrealistic for $\mathrm{Ca}>\mathrm{Ca}_{c}^{*}$ owing to a negative initial curvature, but rather disappears by a bifurcation. Above the critical capillary number, no matching exists between the behavior of the solution at the contact line and the gravity-capillary solution.

\subsection{Film solutions}

A LLD solution can exist whenever there is a positive value $h_{f}$ such that $f\left(h_{f}\right)=0$, which occurs as soon as $\mathrm{Ca}^{*} \geqslant \theta^{*}$. Two major limitations have to be pointed out for these film solutions: first, we might not be able to match this film solution to the hydrostatic region with $h$ remaining positive everywhere. Moreover we restrict our analysis here to an already-established film of zero mass flux, whereas transitory and/or finite flux solutions should be considered (Hocking 2001), and are likely to exist for smaller capillary numbers. Numerically, above the critical capillary number $\mathrm{Ca}_{c}^{*}$, we have always been able to find a zero flux LLD film of thickness $h_{f}$ in the limit $x \rightarrow-\infty$ that could match to the hydrostatic solution without crossing $h=0$ (see Figure 21). Such a solution may be numerically approached, using a special shooting method described in Section 4.1.1. We observed that as the capillary number is decreased, the film surface is shifted down along the $y$-axis (see Figure 6), and we may define a second critical capillary number $\mathrm{Ca}_{c, 2}^{*}$, bellow which the film solution becomes negative in some region. Consequently, if $\mathrm{Ca}_{c, 2}^{*}$ is smaller than $\mathrm{Ca}_{c}^{*}$, hysteresis may occurs, that is, two solutions, a contact line one and LLD film one, may co-exist for same tilt angle and capillary number. 

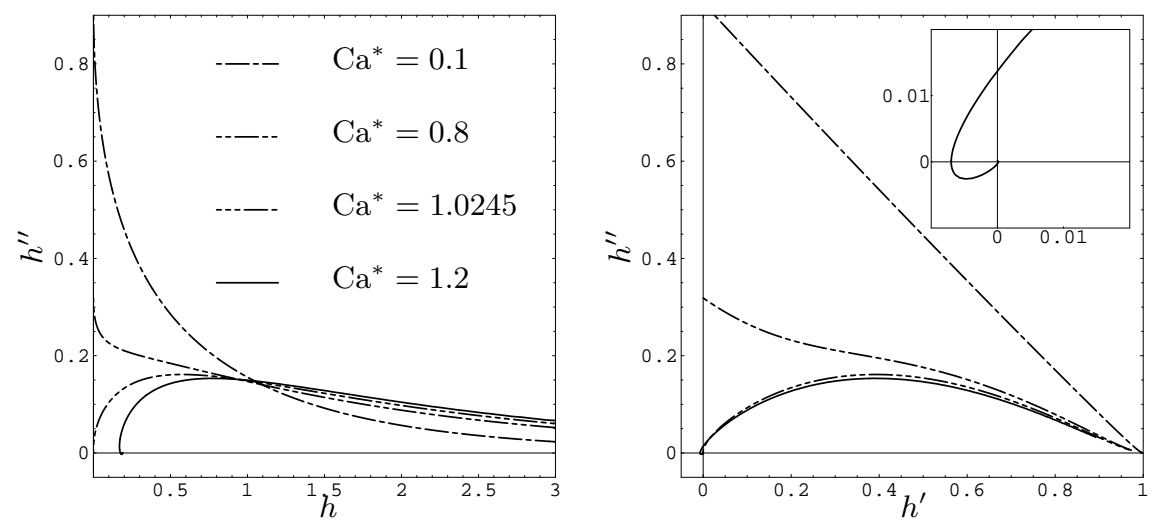

FiguRE 4. Numerical solutions of Equation (2.10) for various capillary numbers, represented in the phase space (projected on the $\left(h, h^{\prime \prime}\right)$ - and $\left(h^{\prime}, h^{\prime \prime}\right)$-planes). The rescaled tilt angle $\theta^{*}$ is fixed to 1 . The trajectories correspond to the physical solutions shown in Figure 2 for subcritical capillary numbers (the three dashed curves), the trajectory starts at a point on the $h^{\prime \prime}$-axis which corresponds to the contact line. On the opposite, the solid line correspond to a film solution, and thus does not cross the $h^{\prime \prime}$-axis. The insert shows the projection of the film solution in the $\left(h^{\prime}, h^{\prime \prime}\right)$-plane, at smaller scale.

\section{Dynamical systems interpretation}

\subsection{Phase space}

In what follow, we interpret and develop the preceding results using dynamical system theory (Strogatz 1994). Let us consider the phase space $\mathcal{V}$ corresponding to Equation (2.10), that is, $\mathbb{R}^{3}$ with coordinates $\left(h, h^{\prime}, h^{\prime \prime}\right)$. Any solution of (2.10) is a trajectory of $\mathcal{V}$, parametrized by $x$, which satisfies

$$
\mathbf{X}^{\prime}=\mathbf{F}(\mathbf{X})=\left(\begin{array}{c}
h^{\prime} \\
h^{\prime \prime} \\
h^{\prime}+f(h)
\end{array}\right) .
$$

Some trajectories (the same as on Figure 21) are represented on Figure 4 Notice that the film solution (solid curve) winds exponentially around a fixed point on the $h$-axis.

\subsubsection{Hydrostatic solutions in the phase space}

For large $x$, following the reasoning of Section 2.5 Equation (4.1) becomes linear:

$$
\mathbf{X}^{\prime}=\left(\begin{array}{lll}
0 & 1 & 0 \\
0 & 0 & 1 \\
0 & 1 & 0
\end{array}\right) \mathbf{X}-\left(\begin{array}{c}
0 \\
0 \\
\theta^{*}
\end{array}\right)
$$

Any solution of (4.2) which satisfies the boundary condition (2.7) is included in a plane called $E_{\infty}$, which may be parameterized by $x$ and the apparent contact angle $\theta_{\text {ap }}^{*}$ introduced in Section 2.5 $E_{\infty}$ is defined by the equation $h^{\prime}-h^{\prime \prime}=\theta^{*}$.

The solutions of the full Equation (4.1) which satisfy (2.7) are included in a twodimensional manifold, called $W$. This manifold tends to $E_{\infty}$ for large $h$. This allows us to approximate numerically the LLD film trajectories, for which we impose boundary conditions at $x \rightarrow-\infty$ and $x \rightarrow+\infty$. We may indeed use a shooting method with initial conditions varying along a constant (large) $h$ line on $E_{\infty}$. The boundary condition at 


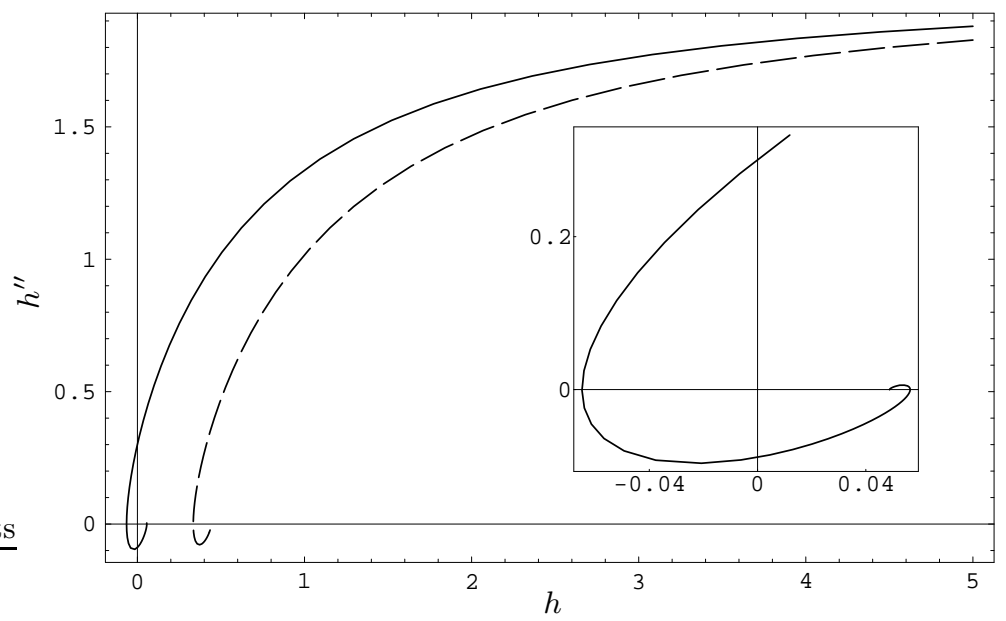

FigURE 5. Intersection of $W$ (the set of trajectories that tend to a horisontal water surface as $x$ tends to $+\infty)$ with the $\left(h, h^{\prime \prime}\right)$ plane. These curves were obtained by a shooting method with $\theta^{*}=2$. Solid line: $\mathrm{Ca}^{*}=2.1$; dashed line: $\mathrm{Ca}^{*}=3$. Insert shows the solid curve at smaller scale.

$x \rightarrow+\infty$ is then approximatively satisfied at any step. The shooting method provides an approximation of the only solution that remains constant as $x$ tends to $-\infty$.

Figure 5 represents the intersection of $W$ with the $\left(h, h^{\prime \prime}\right)$ plane defined by $h^{\prime}=0$, obtained by the shooting method, for two different capillary numbers, above and below $\mathrm{Ca}_{c}^{*}$, for $\theta^{*}=2$. We observe numerically that the major effect of an increase in $\mathrm{Ca}^{*}$ is a translation in the higher $h$ direction. The disappearance of the contact line solution may be described in the following way: any contact line trajectory is embedded in $W$, and the boundary conditions impose that it starts on the $h^{\prime \prime}$-axis, consequently, it can exist only if there is an intersection between $W$ and the $h^{\prime \prime}$-axis. Since the main effect of an increase of $\mathrm{Ca}^{*}$ on $W$ is a translation along the $h$-axis, this intersection disappears above some value $\mathrm{Ca}_{c}^{*}$ of the capillary number. Thus, the existence of a fixed point creates a separatrix on the boundary $W$, that would otherwise be defined over the whole $\left(h, h^{\prime}\right)$-plane, which allows for the sudden disappearance of its intersection with the $h^{\prime \prime}$-axis.

\subsubsection{Fixed points}

A fixed point $\mathbf{X}_{f}$ in phase space corresponds physicaly to a film of constant height $h_{f}$ :

$$
\mathbf{X}_{f}=\left(\begin{array}{c}
0 \\
0 \\
h_{f}
\end{array}\right) .
$$

The existence and values of fixed points depend on the parameters $\theta^{*}$ and $\mathrm{Ca}^{*}$, as presented in Table 1 In the following, we will focus on the largest fixed point $\mathbf{X}_{f}^{+}$, since it is the only one that may be acceptable physically (that is $h_{f}>0$ ). Let us linearize Equation (4.1) around $\mathbf{X}_{f}^{+}$:

$$
\mathbf{X}^{\prime}=\mathbf{J}_{f}^{+}\left(\mathbf{X}-\mathbf{X}_{f}^{+}\right)
$$

where $\mathbf{J}_{f}^{+}$is the jacobian of $\mathbf{F}$ evaluated at $\mathbf{X}_{f}^{+}$, that is 


\begin{tabular}{lc|}
\hline Condition $\mid$ & Fixed points $h_{f} \mid$ \\
\hline$\left|\mathrm{Ca}^{*}<\frac{\theta^{*}}{4}\right|$ & $\emptyset$ \\
\hline $\mathrm{Ca}^{*}=\frac{\theta^{*}}{4} \mid$ & $-\frac{3}{2}$ \\
\hline $\mathrm{Ca}^{*}>\frac{\theta^{*}}{4}\left|\frac{3}{2}\left(-1 \pm \frac{1}{\sqrt{3}} \sqrt{\frac{4 \mathrm{Ca}^{*}}{\theta^{*}}-1}\right)\right|$ \\
\hline
\end{tabular}

TABLE 1. Existence and values of the fixed points of equation (4.1).

\begin{tabular}{|c|}
\hline$\overline{\left|\mathrm{Ca}^{*} \in \Upsilon\left(\theta^{*}\right)\right|} \mid$ \\
$\overline{\left|\mathrm{Ca}^{*} \in \partial \Upsilon\left(\theta^{*}\right)\right|} \mid a, b, c$ \\
$\overline{\left|\mathrm{Ca}^{*} \notin \Upsilon\left(\theta^{*}\right)\right| a,-a / 2,-a / 2+i \Omega,-a / 2-i \Omega \mid}$
\end{tabular}

TABLE 2. Eigenvalues of the jacobian $\mathbf{J}_{f}^{+}$at the largest fixed point. $a, b, c$ and $\Omega$ are real numbers and $\Upsilon\left(\theta^{*}\right)=\left[\frac{9 \theta^{* 3}}{2}\left(1-\sqrt{1-\frac{1}{9 \theta^{* 2}}}\right) ; \frac{9 \theta^{* 3}}{2}\left(1+\sqrt{1-\frac{1}{9 \theta^{* 2}}}\right)\right]$. When the eigenvalues are real, they satisfy: $a<0<b<c$.

$$
\mathbf{J}_{f}^{+}=\left(\begin{array}{ccc}
0 & 1 & 0 \\
0 & 0 & 1 \\
f^{\prime}\left(h_{f}^{+}\right) & 1 & 0
\end{array}\right) .
$$

The local behaviour of solutions around the fixed point depends on the eigenvalues of $\mathbf{J}_{f}^{+}$, which are presented in Table 2 If the eigenvalues are real numbers, one is negative and the two others positive. So there is an unstable manifold of dimension two where the trajectories tend monotonically to the fixed point as $x$ tends to $-\infty$ and a stable manifold $S$ (separatrix) of dimension one. On the other hand, when the two eigenvalues are complex conjugate their common real part is always positive, and the trajectories in the corresponding unstable manifold wind around the fixed point while diverging from it at exponential rate. In physical space, the fluid surface forms damped stationary waves along the plate (see Figure 6). Therefore, the behaviour of $W$ in the vicinity of the fixed point may also be described by this linear expansion. Depending on the parameters, $W$ may either be defined over the whole $\left(h, h^{\prime}\right)$-plane, or tend to the separatrix $S$ (which ends on the fixed point). In the latter case, $W$ winds around the separatrix (as shown in Figure 5] or tends to it monotonically. These various regimes are represented in the diagram of Figure 7 


\subsubsection{Critical capillary number}

At any point on the $h$-axis, $\mathbf{X}^{\prime}$ is parallel to the $h^{\prime \prime}$-axis (see equation (4.1)). Consequently, the intersection of $W$ with the $\left(h, h^{\prime \prime}\right)$ plane (represented on Figure 5) has vertical tangent vectors whenever it crosses the $h$-axis. This explains the behaviour of $h^{\prime \prime}(0)$ close to the critical capillary number (see Figure 3), which may be interpreted as a saddle-node bifurcation. This property is useful for the numerical determination of $\mathrm{Ca}_{c}^{*}$ at a given $\theta^{*}$ : since we know that the second derivative $h^{\prime \prime}(0)$ must vanish at the critical capillary number, we may approximate $\mathrm{Ca}_{c}^{*}$ by a shooting method which varies $\mathrm{Ca}^{*}$ for constant initial conditions (that is, $h=h^{\prime}=h^{\prime \prime}=0$ ). We show in Figure 7 the evolution of the critical capilary number with $\theta^{*}$, together with the diagram showing the different regimes described above. Notice that since $\theta^{*}$ is a rescaled parameter, we have been able to investigate a large range of values, up to $\theta^{*} \approx 10^{7}$.

We did not find any reason for the disappearance of the contact line solution to coincide with the appearance of the LLD film solution as $\mathrm{Ca}^{*}$ is varied. It may well be possible that, as $W$ has already intersected the $h^{\prime \prime}$-axis, the LLD film trajectory rolls up around the fixed point without $h$ ever becoming negative. Some numerical simulations give us confidence that hysteresis indeed occurs (that is $\mathrm{Ca}_{c}^{*}>\mathrm{Ca}_{c, 2}^{*}$ ): a slight hysteresis may indeed be observed in Figure [6 Again, the present study is limited to LLD films of null flux, and other solutions may exist for the same parameters values. Thus, the hysteresis here observed can only describe a reduced part of the solutions set.

When the capillary number is decreased from a supercritical value, the height of the stationary film $h_{f}$ decreases, and eventually the film thickness vanishes at some point $x_{m i n}$ (see Figure 6). This point must be a minimum and in that case both $h\left(x_{\min }\right)$ and $h^{\prime}\left(x_{\min }\right)$ vanish, so this film solution is also a contact line solution. This explains the change of the sign of $h\left(x_{\min }\right)$ observed at point $A$ in Figure [6]

The asymptotic behavior of $\mathrm{Ca}_{c}^{*}$ at large $\theta^{*}$ has also been investigated (Figure 17). We find that the critical capillary number behaves asymptotically as a power law of the tilt angle, that fits to:

$$
\mathrm{Ca}_{c}^{*} \underset{\theta^{*} \rightarrow \infty}{\sim} 0.3936 \theta^{* 1,4998} .
$$

This suggest that for high $\theta^{*}, \mathrm{Ca}_{c}^{*}$ behaves like $\theta^{* 3 / 2}$.

\section{Asymptotic results}

In the following section we describe a rough analytical approach, inspired from that of Eggers (2004a), which leads to the power law (4.3) for the capillary number obtained numerically in the previous section.

\subsection{Overview}

To determine the dependence of the critical capillary number with the angle, we need to better understand how the solution near the contact line connects with the free surface at infinity. We therefore seek to determine the matching between these two domains. This has actually been done when considering the classical plate withdrawal problem. It involves a matching between three regions: one near the contact line, a capillary-viscous one and the gravity-capillary interface (Eggers 2004a). However, two major differences arise in the present case compared to the usual problem. First, equation (3.1) is regular over the whole range $h \in[0 ;+\infty$ [, whereas in the usual problem Navier slip condition (2.3) leads to a pressure divergence at the contact line. However, it has been shown (in the case of an advancing contact line) that the exact form of the slip law near the contact 

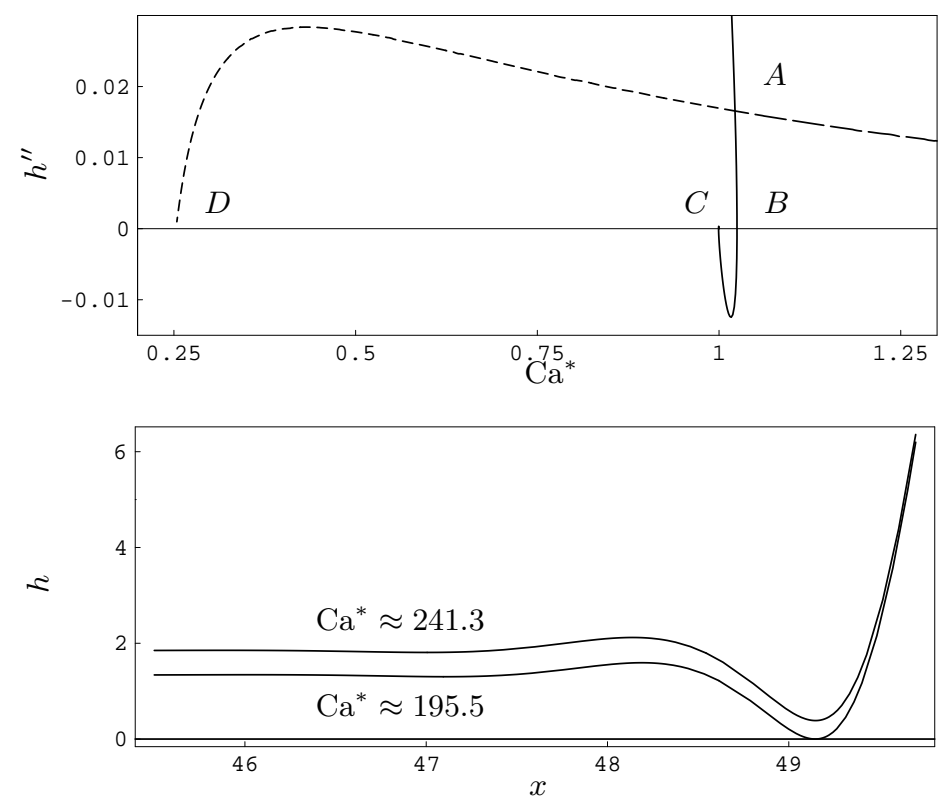

Figure 6. Above: Second derivative of the film height against the capillary number, for $\theta^{*}=1$. The solid line corresponds to $h^{\prime \prime}(0)$ for the contact line solution. The dashed line represents the second derivative of $h$ at the point $x_{\min }$ where in the case of a LLD film solution, the film is thinnest. Large dashes are used if $h\left(x_{\min }\right)>0$, and short dashes otherwise.

Points $A, B, C$ and $D$ correspond respectively to the following values of $\mathrm{Ca}^{*}: \mathrm{Ca}_{c, 2}^{*}, \mathrm{Ca}_{c, 1}^{*}, \theta^{*}$ and $\theta^{*} / 4$.

Below: Two LLD film solutions for $\theta^{*} \approx 66.62$ at different capillary numbers. The curve below correspond to point $A$, since $h\left(x_{\min }\right)$ becomes negative.

line does not influence the matching procedure with the far-field solution of the free surface (Eggers 2004b; Dussan \& Davis 1974). The second difference lies in the contact angle condition that we consider null instead of small but finite for solid plates. Such a condition is crucial as it can be seen from (Eggers 2004a) where the solutions are expanded in powers of the small parameter $\mathrm{Ca} / \theta_{e}^{3}\left(\theta_{e}\right.$ being the static contact angle). Consequently, we cannot obtain a proper matching between the behavior in the contact line zone (cubic polynomial at leading order) with the famous logarithmic behavior in the capillary-viscous region:

$$
h^{\prime}(x)=\left[9 \operatorname{Ca} \ln \left(\frac{\pi}{2^{2 / 3} \beta^{2} x}\right)\right]^{1 / 3}
$$

However, we can bypass this difficulty by a slight change in the equations leading to a single approximation valid over the first two regions (contact line zone and capillaryviscous one).

\subsection{A two-zones matching}

The procedure hereafter presented is based on the assumption that the linear term in the right-hand term denominator of equation (3.1) is not of fundamental importance. In particular, the coefficient $\alpha$ was arbitrarily set to one at the beginning of this study, but numerical investigations have shown that it may be set to much different values, changing the results of only a few percent. For values of $1 / \alpha$ greater than $2 / \sqrt{3}$, equation (3.1) becomes singular for negative values of $h$, which are physically meaningless. We therefore 


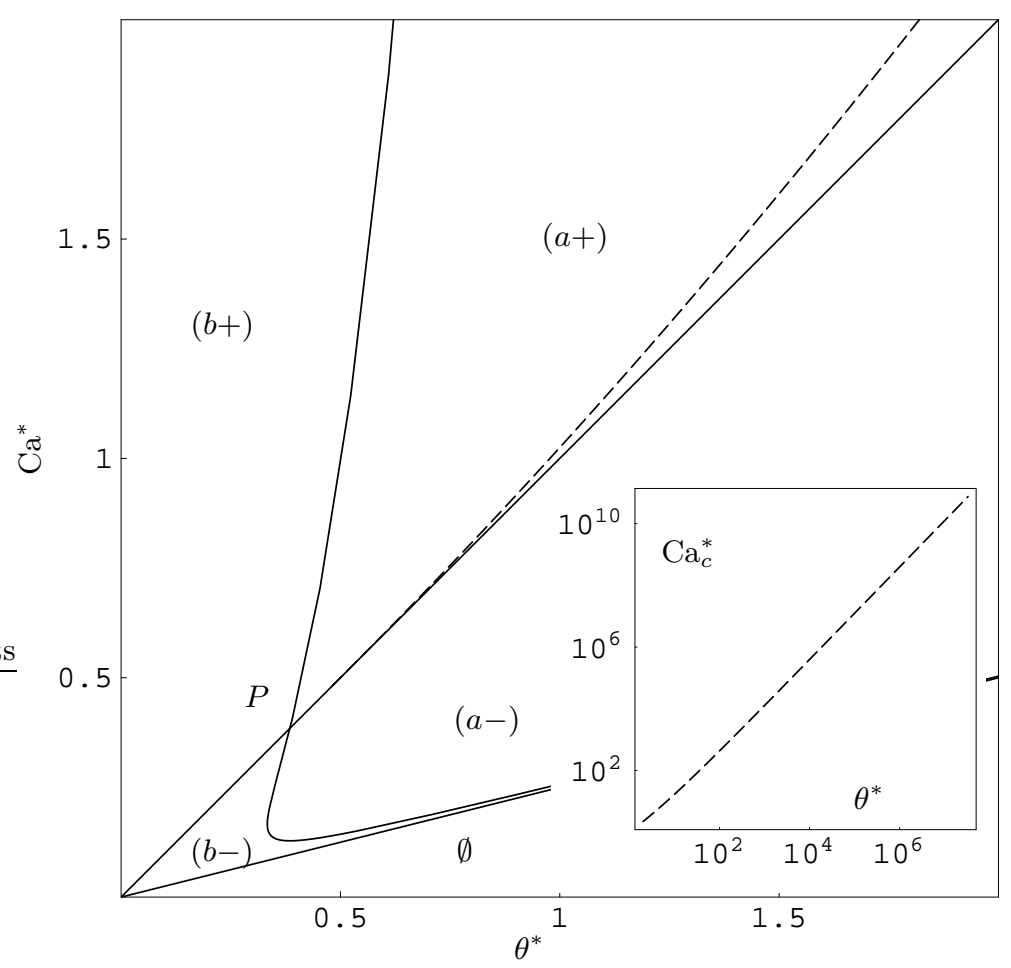

Figure 7. Behaviour of $W$ close to the largest fixed point $\mathbf{J}_{f}^{+}$. $\emptyset$ : no fixed point; + : positive fixed point; -: negative fixed point; $a$ : spiraling trajectories; $b$ : monotonic trajectories. Continuous lines delimit the various behaviors. The dashed line represents the critical capillary number above which the contact line solution disappear $\mathrm{Ca}_{c}^{*}$. Insert: asymptotic behavior of the critical capillary number for large $\theta^{*}$, (logarithmic scale).

set from now on $1 / \alpha=2 / \sqrt{3}$ without any change in the equation properties, so that equation (3.1) reduces to

$$
h^{\prime \prime \prime}-h^{\prime}+\theta^{*}=\frac{\mathrm{Ca}^{*}}{(h / \sqrt{3}+1)^{2}} .
$$

Close enough to the contact line, the film slope $h^{\prime}$ may be neglected due to the boundary condition (3.1). In addition, close to the critical capillary number, $\mathrm{Ca}^{*} \gg \theta^{*}$ (this is suggested by the assymptotic behavior (4.3)), and equation (5.1) becomes

$$
h^{\prime \prime \prime}=\frac{\mathrm{Ca}^{*}}{(h / \sqrt{3}+1)^{2}},
$$

solved analytically (as performed by Duffy \& Wilson (1997)) after the rescaling

$$
x=\frac{\sqrt{3}}{\left(3 \mathrm{Ca}^{*}\right)^{1 / 3}} \xi, h(x)=\sqrt{3}(y(\xi)-1) .
$$

This rescaling leads to Tanner's problem:

$$
y^{\prime \prime \prime}=\frac{1}{y^{2}}, y(0)=1, y^{\prime}(0)=0
$$


Its solution may be parametrized in terms of Airy functions Ai and Bi:

$$
\xi=2^{1 / 3} \frac{\operatorname{Bi}\left(s_{0}\right) \operatorname{Ai}(s)-\operatorname{Bi}(s) \operatorname{Ai}\left(s_{0}\right)}{\operatorname{Bi}^{\prime}\left(s_{0}\right) \operatorname{Ai}(s)-\operatorname{Bi}(s) \operatorname{Ai}^{\prime}\left(s_{0}\right)}, y_{\text {in }}=\frac{1}{\pi^{2}\left(\operatorname{Bi}^{\prime}\left(s_{0}\right) \operatorname{Ai}(s)-\operatorname{Bi}(s) \operatorname{Ai}^{\prime}\left(s_{0}\right)\right)^{2}},
$$

where $s_{0}$ is an integration constant, and $s$ varies between consecutive solutions of equation

$$
\operatorname{Bi}^{\prime}\left(s_{0}\right) \operatorname{Ai}(s)-\operatorname{Bi}(s) \operatorname{Ai}^{\prime}\left(s_{0}\right)=0 .
$$

It was shown in Section 4.1 .3 that, at the critical capillary number, $h^{\prime \prime}$ vanishes (and so does $\left.y^{\prime \prime}\right)$. This property sets $s_{0}$ to zero and the range for $s$ to $\left[s_{1}, 0\left[\right.\right.$, where $s_{1} \approx-1.98635$ is the largest solution to equation (5.3). Matching solution (5.2) with the meniscus solution should provide a condition on $\mathrm{Ca}^{*}$ and $\theta^{*}$. At large $\xi$, the behavior of $y_{\text {in }}$ is

$$
y_{\text {in }}=a \xi^{2}+b \xi+c+O\left(\frac{1}{\xi}\right)
$$

where $a, b$ and $c$ have the following expressions (if we define $z(s)=\pi(\operatorname{Bi}(0) \operatorname{Ai}(s)-$ $\operatorname{Bi}(s) \operatorname{Ai}(0)))$ :

$$
\begin{gathered}
a=\left(\frac{\operatorname{Bi}^{\prime}(0)}{2^{1 / 3} \operatorname{Bi}\left(s_{1}\right)}\right)^{2} \approx 0.758947, b=2 a\left(\frac{2^{1 / 3} \mathrm{Bi}^{\prime}\left(s_{1}\right)}{\operatorname{Bi}^{\prime}(0) z^{\prime}\left(s_{1}\right)}\right) \approx 1.12697, \\
c=\frac{b^{2}}{4 a}+\frac{\operatorname{Bi}^{\prime}\left(s_{1}\right) z^{\prime \prime}\left(s_{1}\right)-\operatorname{Bi}^{\prime \prime}\left(s_{1}\right) z^{\prime}\left(s_{1}\right)}{\operatorname{Bi}\left(s_{1}\right) z^{\prime 3}\left(s_{1}\right)} \approx 2.06713 .
\end{gathered}
$$

Matching $y_{\text {in }}$ with the second-order Taylor expansion of $h_{\infty}$ for vanishing $x$ leads to:

$$
\theta^{*}-\theta_{\mathrm{ap}}^{*}=2 a 3^{1 / 6} \mathrm{Ca}_{c}^{* 2 / 3}, A_{\infty}=\sqrt{3}(c-1)-\theta^{*}+\theta_{\mathrm{ap}}^{*}, \theta_{\mathrm{ap}}^{*}=b\left(3 \mathrm{Ca}_{c}^{*}\right)^{1 / 3} .
$$

When the critical capillary number tends to infinity, the first of the previous equations reads

$$
\mathrm{Ca}_{c}^{*} \underset{\theta^{*} \rightarrow \infty}{\sim} \frac{1}{(2 a)^{3 / 2} 3^{1 / 4}} \theta^{* 3 / 2} .
$$

This fits remarkably well with the numerical estimation (4.3), since $1 /\left((2 a)^{3 / 2} 3^{1 / 4}\right) \approx$ 0.40631 (the above numerical fit giving 0.3936). The matching is compared to numerical results in Figure 8 for a high value of $\theta^{*}$, and we observe a remarkable agreement with a reasonably large overlap region.

Moreover, this matching procedure provides the following law for the apparent contact angle, at the critical speed: $\theta_{\mathrm{ap}}=b\left(3 \mathrm{Ca}_{c}\right)^{1 / 3}$ (notice that the rescaling term $\lambda$ disappears). This is reminiscent of the famous Tanner law which relates the contact line velocity to the apparent contact angle (Tanner 1979).

\section{Discussion and conclusions}

In this work, a continuum model of the forced dewetting on a porous material has been presented. In the framework of lubrication, an ordinary non-linear differential equation was derived, close to the one investigated by Hocking (2001). Even if the (microsopic) contact angle is assumed to vanish, a stationary contact line is found to exist for low dewetting velocity. Moreover, a transition between this steady contact line and the deposit 


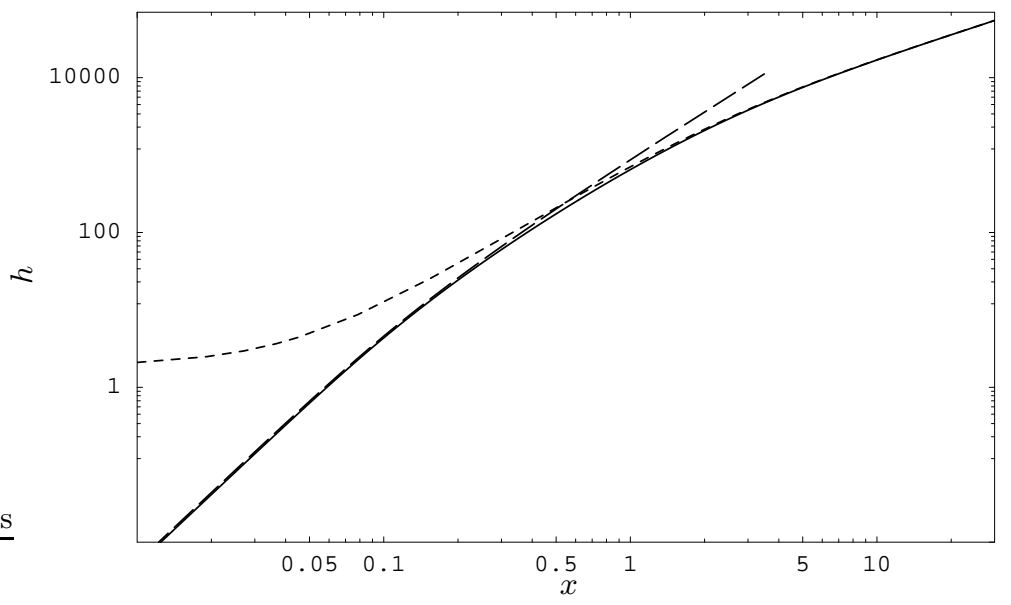

Figure 8. Comparison between numerical results (solid line) and the matching presented in this paper (long and short dashes), for $\theta^{*} \approx 1886.19$ and $\mathrm{Ca}^{*} \approx \mathrm{Ca}_{c}^{*} \approx 32871.1$, in logarithmic scales. Long dahes: analytical solution to Tanner's problem; short dashes: capillary-gravity meniscus.

of a LLD-film must occur, since there is a critical capillary number above which no contact line solution can exist.

In the present study, the apparent contact angle (as defined by Eggers (2004a), that is $\theta_{\mathrm{ap}}^{*} \lambda$ in the present notations), does not vanish at the critical capillary number (see Section 51). This behavior is different from the one obtained by Eggers (2004a), where the apparent contact angle was found to vanish at the transition from a contact line to a LLD film in the case of finite microscopic contact angle $\theta_{e}$. This discrepancy is surprising since one would expect the problem studied by Eggers (2004a) to correspond with the present one when $\theta_{e} \rightarrow 0$ and $\lambda \rightarrow 0$. However, in the latter limit the rescaling by $\lambda$ used here cannot hold. Also, as previously mentioned, the expansion in powers of $\mathrm{Ca} / \theta_{e}^{3}$ performed by Eggers (2004a) becomes ill-defined.

Different conclusions can be drawn from our results regarding the erosion experiment performed by Daerr et al. (2003). First, the existence diagram of the contact line can be drawn using the experimental values of the physical parameters. In the present theory, the permeability $k$ of the porous material is crucial, as is the characteristic slip length at the solid-liquid interface. The value of this parameter may depend strongly on the compaction of the granular material (say between $10^{-12} \mathrm{~m}^{2}$ and $900.10^{-12} \mathrm{~m}^{2}$, respectively the value measured by Daerr et al. (2003) and the square of the grain size). Figure 9 presents the critical velocities obtained for these two extremal values of the permeability. For the lowest permeability, and down to the smallest withdrawal velocities of the erodible plate, no contact line can exist. On the other hand, when choosing the largest permeability, the critical speed line is of order of those of the experiement.

The flow acts on the granular medium mainly through the bottom shear rate $\tau=$ $\partial u / \partial y$, which is known to trigger the erosion process (see Charru et al. (2004)). From this shear rate, we can define the Shields number $S$, which compares the viscous force applied to the grains by the flow, to gravity force:

$$
S=\frac{\eta \tau}{\left(\rho_{g}-\rho\right) g d}
$$

where $\rho_{g}$ is the density of the grains (this expression stands only for small angles). Though the erosion prossess on a granular bed results from discrete and complex phe- 


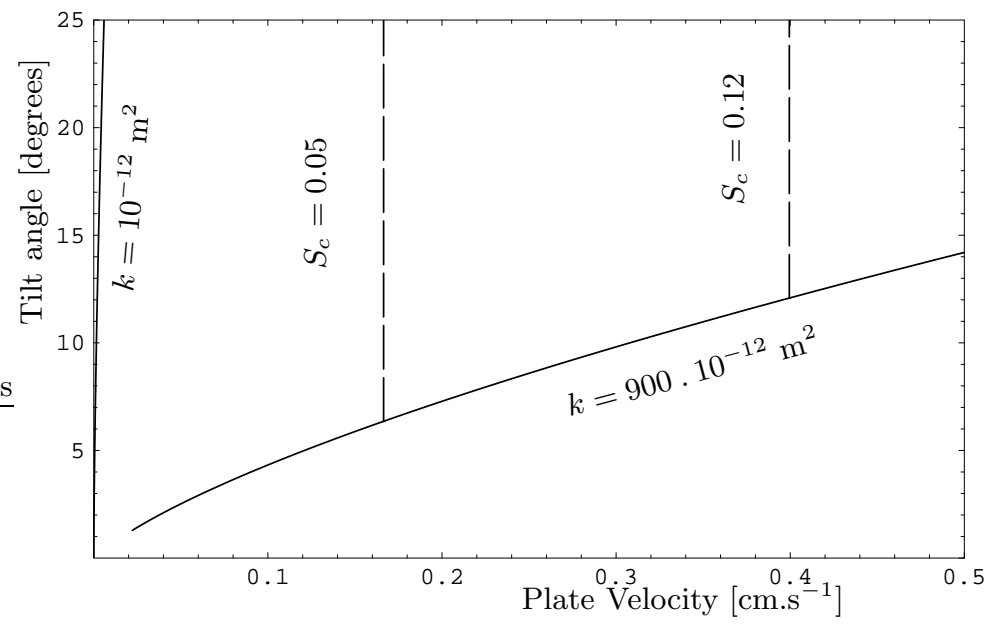

FiguRE 9. Critical speed for two values of the permability $k$. For each value of the permeabiblity, a contact line can exist only above the corresponding solid curve. The dashed line represents constant Shields numbers $S_{c}$. The values of the physical parameters used in this model are those of Daer et al. (Daerr et al. 2003): $g=9.81 \mathrm{~m} . \mathrm{s}^{-2}, \rho=1000 \mathrm{~kg} \cdot \mathrm{m}^{-3}, \rho_{g}=2750 \mathrm{~kg} . \mathrm{m}^{-3}$, $\eta=10^{-3} \mathrm{~kg} \cdot \mathrm{m}^{-1} \cdot \mathrm{s}^{-1}$ and $\gamma_{s}=0.07 \mathrm{~N} . \mathrm{m}^{-1}$. The velocity and inclination ranges are those of the experiment.

nomena, classical models assume that it starts at a threshold value of the shear rate, at which a critical Shields number $S_{c}$ is defined (Charru et al. 2004). A typical value for $S_{c}$ is 0.05 (see among others Fredsoe \& Deigaard (1992)), but Daerr et al. (2003) used $S_{c}=0.12$ to fit their data. In addition to this large range of possible values, note that the critical Shields number is a function of the slope of the bottom: the more inclined it is, the easier it is for the flow to lift grains, thus the tilt reduces the value of $S_{c}$. The shear rate may be deduced from our model, as a function of the dimensionless height of the film $h$ :

$$
\tau=\frac{U h}{\sqrt{k}\left(h^{2} / 3+h+1\right)} .
$$

This expression admits a maximum value $\tau_{\max }=U /(\sqrt{k}(1+2 / \sqrt{3}))$ for $h=\sqrt{3}$, that is necessarily reached in the case of a contact line, since $h$ stretches from zero to infinity. If the Shields number is assumed to be independent of the tilt angle of the plate (to first order), $S=S_{c}$ defines a vertical line in Figure 9 (represented only for $k=900.10^{-12} \mathrm{~m}^{2}$ ). On the left of such a line, no erosion should occur since the Shields number is smaller than the critical value, thus to account for the erosion patterns observed by Daerr et al. (2003) at small velocities, a low value of $S_{c}$ is required.

Now, if a LLD film covers the plate, $h$ admit a minimum value $h_{m i n}$ which is found numerically to increase with the plate velocity. Once $h_{\text {min }}$ is larger than $h=\sqrt{3}$, the maximum value of the shear rate becomes smaller than $\tau_{\max }$, and could decrease as the capillary number increases. Thus, the transition from contact line to LLD film could induce a strong change in the stress regime. If the physical parameters are choosen in the experimental range, no stress jump is observed numerically at the transition, but two assumptions should be relaxed in order to evaluate precisely the bottom stress close to the critical capillary number: the permanent regime, and the constraint of null outflow $(Q=0)$. In particular, if a negative outflow appears in reality (that is, if water is withdrawn from the bath), the shear rate should be reduced. Such a sharp stress 
variation could provide an explanation for the transition between the different erosion patterns observed by Daerr et al. (2003).

At the transition from contact line to LLD film, transient regimes should not be ignored. They have been studied in the litterature for non-vanishing contact angles (Hocking 2001), and future studies will aim at understanding the case of null contact angle, which has been shown here to be quite different.

It is our pleasure to thank Daniel Lhuillier, Pierre-Yves Lagrée, Eric Clément, Florent Malloggi and Jens Eggers for stimulating discussion.

\section{Appendix A. Pressure divergence at the contact line}

We aim here to demonstrate briefly that the classical Navier slip condition, leading to Equation (2.3), is not sufficient to eliminate all the singularities at the contact-line, even for a non-vanishing microscopic contact angle $\left(\theta_{e}>0\right)$. Indeed, the first order expansion of Equation (2.3) is (Eggers 2004a; Hocking 1983)

$$
h^{\prime}(x) \sim \theta_{e}-\frac{3 \mathrm{Ca}}{\theta_{e}^{2}}\left(1+\ln \left(\frac{x \theta_{e}}{\lambda_{N}}\right)\right),
$$

where $h$ is dimensionless but not rescaled (that is, $\mathrm{h}$ is the height of the water surface divied by the capillary length). In the lubrication approximation that we have used throughout, the pressure at the plate reads

$$
\left.p\right|_{y=0}=\rho g \cos (\theta) h-\gamma h^{\prime \prime},
$$

and since $h^{\prime \prime}(x) \sim-3 \mathrm{Ca} /\left(\theta_{e}^{2} x\right)$ the pressure diverges at the contact line. In the present paper, due to the permeability of the porous plate, the expansion of $h$ near the contactline is a third order polynomial $\left(h \sim h^{\prime \prime}(0) x^{2} / 2+\left(\mathrm{Ca}^{*}-\theta^{*}\right) x^{3} / 6\right)$, and thus the pressure does not diverge.

\section{Appendix B. Derivation of the fundamental equation}

In the following, we aim to derive Equation (2.6) from the two-dimensional NavierStokes equation. $x$ and $y$ refer to the axes of Figure 1 In the frame of the lubrication approximation, and assuming both a permanent regime and small Reynolds number, momentum conservation reads

$$
\begin{gathered}
-\frac{1}{\rho} \frac{\partial p}{\partial x}+g \sin (\theta)+\nu \frac{\partial^{2} u}{\partial y^{2}}=0 \\
-\frac{1}{\rho} \frac{\partial p}{\partial y}-g \cos (\theta)=0,
\end{gathered}
$$

where $u$ and $v$ stand for the water velocity components respectively parallel and perpendicular to the plate. The second equation may be integrated to give

$$
p=\rho g \cos (\theta)(h-y)+p_{L}
$$

where $p_{L}$ is the pressure due to surface tension. Now, if $\theta$ is small enough, the slope $h^{\prime}$ of the free surface should remain reasonably small, so that $h^{\prime \prime}$ approximates its curvature, and $p_{L} \approx-\gamma h^{\prime \prime}$ (and, similarly, $\sin (\theta) \approx \theta$ and $\cos (\theta) \approx 1$ at first order). The boundary 
conditions on $u$ at the bottom and the top of the film are (see Equations (2.1), (2.4) and (2.5)

$$
\begin{gathered}
\left.u\right|_{y=0}+U=\left.\frac{\sqrt{k}}{\alpha} \frac{\partial u}{\partial y}\right|_{y=0}-\frac{k}{\eta}\left(\left.\frac{\partial p}{\partial x}\right|_{y=0}-\rho g \theta\right) \\
\left.\frac{\partial u}{\partial y}\right|_{y=h(x)}=0
\end{gathered}
$$

thus integrating Equation (B1) we obtain

$$
u=\frac{1}{\nu}\left(\frac{1}{\rho} \frac{\partial p}{\partial x}-g \theta\right)\left(\frac{y^{2}}{2}-h y-\frac{\sqrt{k}}{\alpha} h-k\right)+U .
$$

The mass flux is

$$
\rho \int_{0}^{h} u d y=Q
$$

In steady state, mass conservation imposes that $Q$ is a constant which vanishes in the case of a contact line. This condition provides the non-linear equation studied throughout this paper: $Q=0$ reads

$$
\frac{\gamma}{\rho} h^{\prime \prime \prime}-g h^{\prime}+g \theta=\frac{\nu U}{h^{2} / 3+h \sqrt{k} / \alpha+k}
$$

which, after rescaling $x$ and $h$ by the capillary length $l_{c}$, reduces to Equation (2.6).

\section{REFERENCES}

Aradian, A., Raphä̈L, E. \& De Gennes, P.-G. 2000 Dewetting on porous media with aspiration. The European Physical Journal E 2, 367-376.

BACRI, L. \& BRochARD-WyART, F. 2001 Dewetting on porous media. Europhysics Letters 56 (3), 414-419.

Beavers, S. \& Joseph, D. 1967 Boundary condition at a naturally permeable wall. Journal of Fluid Mechanics 30, 197-207.

Blake, T. \& Ruschak, K. 1979 A maximum speed of wetting. Nature 282, 489-491.

Charru, F., Mouilleron, H. \& Eiff, O. 2004 Erosion and deposition of particles on a bed sheared by a viscous flow. J. Fluid Mech. 519, 55-80.

Daerr, A., Lee, P., Lanuza, J. \& Clément, É. 2003 Erosion patterns in a sediment layer. Physical Review E 67.

DeRJAGin, B. 1943 On the thickness of a layer of liquid remaining on the walls of vessels after their emptying, and the theory of the application of photoemulsion after coating on the cine film. Acta physico-chemica USSR 20, 349.

Duffy, B. \& Wilson, S. 1997 A third order differnetial equation arising in thin-film flows and relevent to tanner's law. Appl. Math. Lett. 10 (3), 63-68.

Dussan, V. E. \& Davis, S. 1974 On the motion of a fluid-fluid interface along a solid surface. J. Fluid Mech. 65, 71.

Eggers, J. 2004a Hydrodynamic theory of forced dewetting. Physical Review Letters 93 (9), 3491-3494.

EgGers, J. $2004 b$ Toward a description of contact line motion at higher capillary numbers. Physics of Fluids 16 (9), 3491-3494.

Fredsoe, J. \& DeigaArd, R. 1992 Mechanics of coastal sediment transport. World Scientific. De Gennes, P.-G. 1984 Dynamique d'étalement d'une goutte. C. R. Acad. Sc. Paris 298 (4), 111-115. 
DE Gennes, P.-G. 1985 Wetting: statics and dynamics. Reviews of modern physics 57 (3), 827-861.

Goyeau, B., Lhuillier, D., Gobin, D. \& Velarde, M. 2003 Momentum transport at a fluid-porous interface. International Journal of Heat and Mass Transfer 46, 4071-4081.

Hadjiconstantinou, N. G. 2003 Comment on cercignani's second-order slip coefficient. Phys. Fluids 15 (8), 2352-2354.

Hervet, H. \& De Gennes, P.-G. 1984 Dynamique du mouillage: film précurseur sur solide "sec". C. R. Acad. Sc. Paris 299 (9), 499-503.

Hocking, L. 1983 The spreading of a thin drop by gravity and capillarity. Q. J. Mech. Appl.Math. 36, 55-69.

Hocking, L. 2001 Meniscus draw-up and draining. Euro. Jnl of Applied Mathematics 12, 195208.

Huh, E. \& Scriven, L. 1971 Hydrodynamic model of steady movement of a solid/liquid/fluid contact line. J. Colloid Interface Sci. 35 (1), 85-101.

Landau, L. \& Levich, B. 1942 Dragging of a liquid by a moving plate. Acta physico-chemica USSR 17, 42.

Manneville, P. 1990 Dissipative Structures and Weak Turbulence. Academic Press.

Maurer, J., Tabeling, P., Joseph, P. \& Willaime, H. 2003 Second-order slip laws in microchannels for helium and nitrogen. Phys. Fluids 15 (9), 2613-2621.

Neale, G. \& NADER, W. 1974 Practical significance of brinkman's extension of darcy's law. The Canadian Journal of Chemical Engineering 52, 475-478.

Raphä̈L, É. \& De Gennes, P.-G. 1999 Imprégnation d'un ruban poreux. C. R. Acad. Sci. Paris 327, 685-689.

Renardy, M., Renardy, Y. \& Li, J. 2001 Numerical simulation of moving contact line using a volume-of-fluid method. J. Comput. Phys. 171, 243-263.

Scherer, M., Melo, F. \& Marder, M. 1999 Sand ripples in an oscillating annular sand-water cell. Phys. Fluids 11 (1), 58.

Schorghofer, N., Jensen, B., Kudrolli, A. \& Rothman, D. H. 2004 Spontaneous channelization in permeable ground: theory, experiment, and observation. J. Fluid Mech. 503, $357-374$

Seppecher, P. 1996 Moving contact lines in the cahn-hilliard theory. Int. J. Engng Sci. 34 (9), 977-992.

Stegner, A. \& Wesfreid, J. 1999 Dynamical evolution of sand ripples under water. Phys. Rev. E 60, R3487.

Strogatz, S. 1994 Nonlinear dynamics and chaos. Addison-Wesley.

TAnner, L. 1979 The spreading of silicone oil drops on horizontal surfaces. J. Phys. D12, $1473-1480$. 